\title{
Phytopathology

\section{A Bayesian Approach to Meta-Analysis of Plant Pathology Studies}

\author{
A. L. Mila and H. K. Ngugi
}

First author: Department of Plant Pathology, North Carolina State University, Campus Box 7405, Raleigh 27606; and second author: Department of Plant Pathology, Pennsylvania State University, Fruit Research \& Extension Center, Biglerville 17307.

ABSTRACT

\begin{abstract}
Mila, A. L., and Ngugi, H. K. 2011. A Bayesian approach to metaanalysis of plant pathology studies. Phytopathology 101:42-51.

Bayesian statistical methods are used for meta-analysis in many disciplines, including medicine, molecular biology, and engineering, but have not yet been applied for quantitative synthesis of plant pathology studies. In this paper, we illustrate the key concepts of Bayesian statistics and outline the differences between Bayesian and classical (frequentist) methods in the way parameters describing population attributes are considered. We then describe a Bayesian approach to meta-analysis and present a plant pathological example based on studies evaluating the efficacy of plant protection products that induce systemic acquired resistance for the management of fire blight of apple. In a simple random-effects model assuming a normal distribution of effect sizes and no prior information (i.e., a noninformative prior), the results of the Bayesian meta-analysis are similar to those obtained with classical methods. Implementing the same model with a Student's $t$ distribution and a noninformative prior for the effect sizes, instead of a normal distribution, yields similar results for all but acibenzolar-S-methyl (Actigard) which was evaluated only in seven studies in this example. Whereas both the
\end{abstract}

classical $(P=0.28)$ and the Bayesian analysis with a noninformative prior $(95 \%$ credibility interval $[\mathrm{CRI}]$ for the log response ratio: -0.63 to 0.08 ) indicate a nonsignificant effect for Actigard, specifying a $t$ distribution resulted in a significant, albeit variable, effect for this product (CRI: -0.73 to -0.10 ). These results confirm the sensitivity of the analytical outcome (i.e., the posterior distribution) to the choice of prior in Bayesian meta-analyses involving a limited number of studies. We review some pertinent literature on more advanced topics, including modeling of among-study heterogeneity, publication bias, analyses involving a limited number of studies, and methods for dealing with missing data, and show how these issues can be approached in a Bayesian framework. Bayesian meta-analysis can readily include information not easily incorporated in classical methods, and allow for a full evaluation of competing models. Given the power and flexibility of Bayesian methods, we expect them to become widely adopted for meta-analysis of plant pathology studies.

Additional keywords: acibenzolar-S-methyl, Apogee, Erwinia amylovora, harpin protein, Messenger, prohexadione-calcium, systemic acquired resistance.
An important goal in statistical analyses is to draw inference about unobserved quantities. Within a classical (frequentist) statistics framework, inferences often take the form of point estimates of unknown parameters, and uncertainty about the true parameter value is represented with confidence intervals $(5,14)$. Within the Bayesian framework, parameters (and not just their estimates) are treated as random variables and described with probability distributions rather than point values $(5,8)$ (Table 1 ). The probabilities are interpreted as 'degrees of belief' that are subject to one's knowledge about the quantity being described,

Corresponding author: H. K. Ngugi; E-mail address: hkn3@psu.edu

doi:10.1094/PHYTO-03-10-0070

(C) 2011 The American Phytopathological Society and which can be updated as more information (data) becomes available. There are some fundamental differences between the classical and Bayesian approaches to statistics, including in the inference that can be drawn from analysis of the same data set, that are beyond the scope of this paper. Table 1 and Bolstad (5), summarizes some of the major differences between classical and Bayesian statistics in general. Each approach has its advantages and disadvantages but these are sometimes controversial and are not discussed in detail here. In our view, the choice between a Bayesian and a classical approach to meta-analysis is mostly one of practical convenience; for a given data set and the same a priori assumptions, both approaches typically yield numerically similar results, although Bayesian analyses often indicate wider confidence intervals for the mean effects than the classical methods in models assuming among-study variability (31). Numerically dif- 
ferent results occur when the Bayesian approach allows incorporation of information that otherwise cannot be readily included in a classical meta-analysis, for example, through the use of informative priors as illustrated below.

\section{BACKGROUND ON BAYESIAN STATISTICS}

Mathematical formulation and definitions. In Bayesian analysis, there are three key components: prior knowledge or experience, current data, and probability of outcome (Fig. 1). In statistical terminology, these concepts are specified as the prior

TABLE 1

Differences between Bayesian and classical statistics

\begin{tabular}{|c|c|}
\hline Bayesian & Classical \\
\hline $\begin{array}{l}\text { Paradigm focuses on the } \\
\text { plausibility of an event given } \\
\text { incomplete knowledge. }\end{array}$ & $\begin{array}{l}\text { Long-run expected frequency of } \\
\text { occurrence. }\end{array}$ \\
\hline $\begin{array}{l}\text { Parameters, the numerical } \\
\text { attributes of a population, are } \\
\text { random variables. }\end{array}$ & $\begin{array}{l}\text { Parameters are fixed but } \\
\text { unknown constants. }\end{array}$ \\
\hline $\begin{array}{l}\text { Inference is based on a credible } \\
\text { interval (CRI), centered near } \\
\text { the sample mean, but } \\
\text { tempered by prior beliefs } \\
\text { concerning the mean. }\end{array}$ & $\begin{array}{l}\text { Inference is based on confidence } \\
\text { interval (Cl), centered at the } \\
\text { sample mean. }\end{array}$ \\
\hline $\begin{array}{l}\text { Because probability statements } \\
\text { are only allowed for random } \\
\text { variables, the CRI has a direct } \\
\text { probability interpretation. The } \\
95 \% \text { CRI is interpreted as: } \\
\text { there is a } 95 \% \text { probability that } \\
\text { this interval contains the } \\
\text { mean. }\end{array}$ & $\begin{array}{l}\text { True mean may or may not be } \\
\text { within the } 95 \% \mathrm{Cl} \text {. That is, the } \\
\text { true mean, being a single fixed } \\
\text { value, does not have a } \\
\text { distribution. The sample mean } \\
\text { (based on long-run sampling) } \\
\text { has a distribution. The } 95 \% \mathrm{Cl} \text {, } \\
\text { therefore, is interpreted as: } \\
95 \% \text { of similar intervals would } \\
\text { contain the true mean, if each } \\
\text { interval were constructed from } \\
\text { a different random sample. }\end{array}$ \\
\hline
\end{tabular}

distribution, the likelihood function, and the posterior distribution. These three components are formally combined by Bayes rule to estimate parameters:

$$
P(\theta \mid y)=\frac{P(\theta) \cdot P(y \mid \theta)}{P(y)} \propto P(\theta) \cdot P(y \mid \theta)
$$

where $P(\bullet)$ is a probability and $P(\bullet \bullet \bullet)$ is a conditional probability. The symbol $\propto$ denotes proportionality, and $\theta$ is a set (i.e., vector) of parameters to be estimated (8). $P(\theta)$ is the prior probability, and $P(y \mid \theta)$ is the probability of the observations given the parameter vector; the latter probability is known also as the likelihood function. From the likelihood function and the prior, the posterior probability of the parameters given the data is determined from equation 1 as $P(\theta \mid y)$. The distributional results for a collection of $\boldsymbol{y}$ values follow directly from these individual probabilities based on rules of conditional probability as described in Bolstad (5).

Existing information (or opinion) about the parameters of interest $(\boldsymbol{\theta})$ is summarized quantitatively via probability distributions referred to as prior distributions or simply priors (Fig. 1). Specifically, the prior distribution is a probability statement that summarizes our current beliefs about an event before data have been collected. By definition, these prior beliefs are subjective, hence the term degrees of belief.

In biological research fields such as plant pathology, a Bayesian approach can cause some difficulties for investigators because they are not accustomed to representing their prior knowledge about a treatment effect in the form of a probability distribution. In addition, there may be very little prior knowledge about new experimental treatments, so investigators may be reluctant, or unable, to quantify their prior beliefs about the treatments' performance. The choice of a prior distribution can also be very controversial (31). Different investigators may select different priors for the same situation, which could lead to different conclusions about the trial. This is especially true when the data are based on a small sample size because in such situations, the prior distributions are modified only slightly by the data to form the posterior distributions (29). This tends to weight the posterior distribution very closely to the prior; that is, the results are based almost entirely on one's prior assumptions or experience. We demon-
FIGURE 1

Flowchart representing associations between the components of Bayesian statistics. Bold text indicates the three key components in equation 1 (i.e., prior information, current data, and probability of outcome), whereas text in italics represents probability components such as prior distributions.

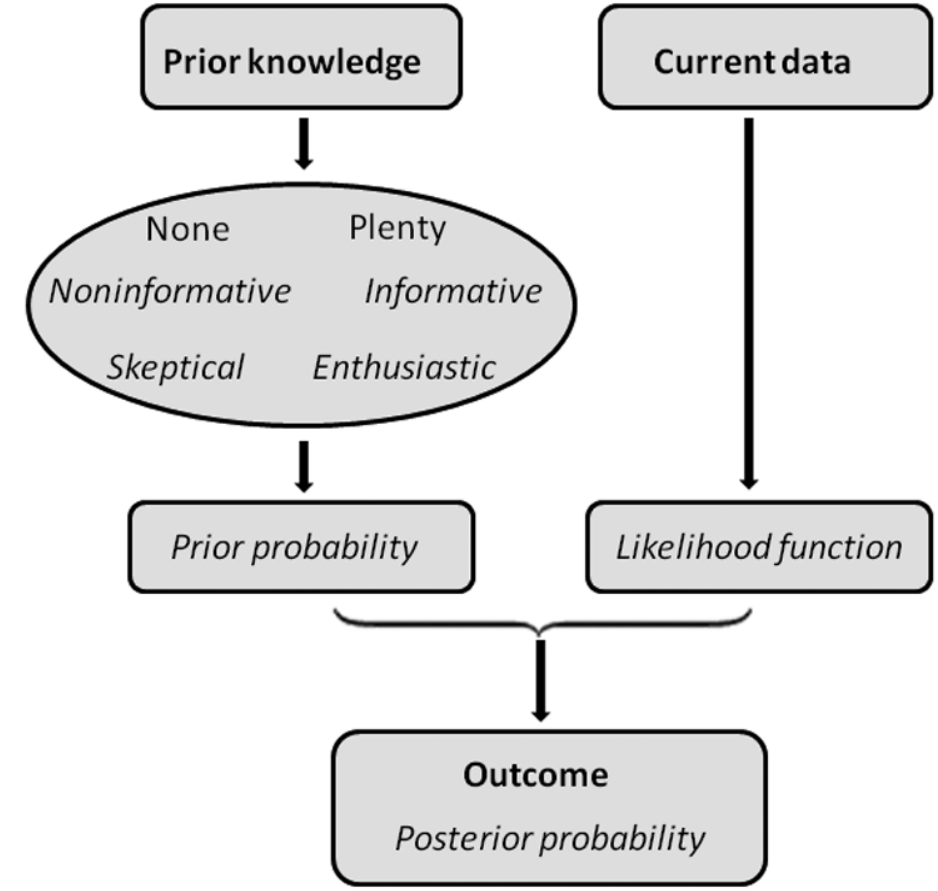

Vol. 101, No. 1, 2011 
strate the influence of sample size on posterior distribution with an example from plant pathology later in this paper.

Depending on the level of existing evidence about the parameters $\theta$, a prior distribution may be noninformative (or vague) when no evidence is available, informative when some evidence is available, or very informative when strong evidence is available (Fig. 1) (8). When there is little prior information to base one's assumptions of the distribution on, Bayesians employ a reference (or vague or noninformative) prior which is intended to represent a minimal or zero amount of prior information (Fig. 2). Although vague priors may yield results similar to those of a classical approach, the priors may be unrealistic because they tend to assign equal weight to all values of $\bullet$. Similarly, skeptical prior distributions are those that quantify the belief that large treatment effects are unlikely; i.e., it is questionable that a treatment is beneficial (11). Conversely, enthusiastic prior distributions are those that quantify large treatment effects and express the initial belief that the treatment is very effective. Alternatively, a prior distribution may be based on previous studies and therefore is adjusted and/or weighted to account for potential biases (Fig. 2). In medical trials, for example, subjective clinical opinions may form the basis of a prior distribution (subjective prior) (22). Elicitation of opinion can be carried out using techniques such as interviews, questionnaires or interactive computer packages, literature searches, or preliminary surveys (13). These are not mutually exclusive; for example, subjective judgment about relevance or changed circumstances may be needed to modify results from an objective meta-analysis (22).

Classical meta-analyses make the assumption that the studies under review are a random sample from a hypothetical population of possible studies $(16,31)$, but this assumption is not always true, for example owing to publication bias. Nonetheless, this assump- tion provides a rationale for using a noninformative prior in the absence of previous knowledge or experience when carrying out Bayesian meta-analyses of plant pathology studies.

The posterior distribution describes the new state of knowledge about the parameters $\theta$ in light of the experience gained. Its shape depends on the prior and likelihood distributions, but whenever the latter distributions are both assumed to be normal, the resulting posterior distribution is also normal. The posterior distribution can be used to estimate a mean, median, and a 2.5th and 97.5th percentile probability interval (also known as a 95\% credible interval, CRI), which infers that the Bayesian is $95 \%$ certain that $\theta$ lies within this interval $(5,8,31)$. Also, it is often useful to give the probability that an effect is in a particular region, for example, the probability that the log of the odds ratio is smaller than -0.5 . It should be noted that because the posterior distribution incorporates information from a subjective prior distribution, it is also subjective $(5,31)$.

Bayesian methods and meta-analysis: "a natural fit". Researchers commonly perform trials that are analyzed individually and their contribution to accruing knowledge is then assessed informally. However, meta-analysis is increasingly used to combine the information from similar trials into a formal quantitative summary $(16,18)$. Among the reasons for performing a metaanalysis are questions commonly raised by researchers such as: "What are we going to gain from the results of this trial in the light of the information known before the trial is conducted?" or "What do we think about the relative benefit of the treatments before knowing the results of the current trial?" In the Bayesian context, these questions are easily rephrased as: "What is the previous evidence on the relative benefits of treatment?" "What is the current evidence from previous trials?" and "What is the updated evidence, once we combine the previous evidence with
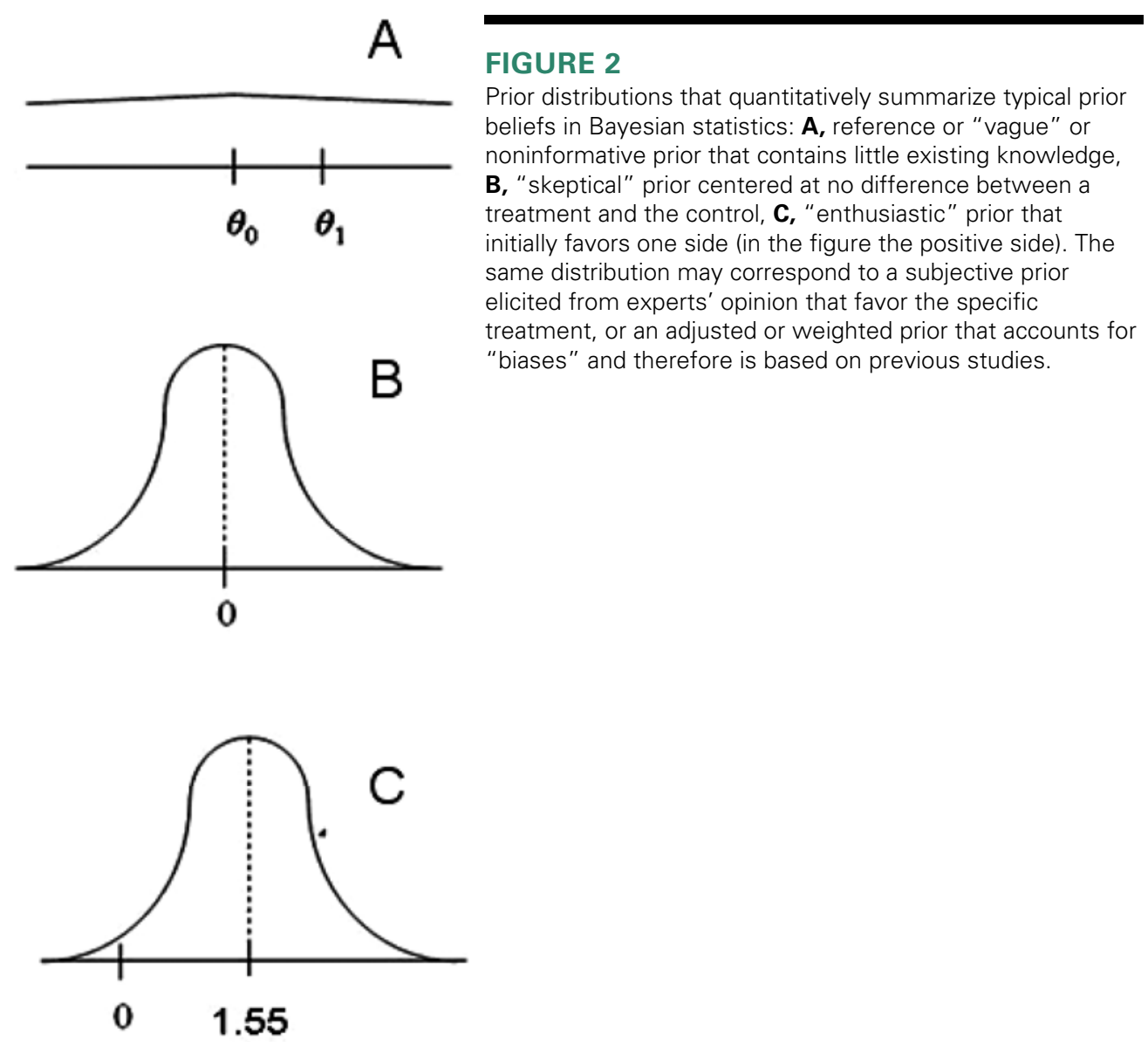
the new evidence?" The concept of updating beliefs or evidence is the essence of Bayesian statistics and, therefore, their implementation in meta-analysis comes as a natural fit (22).

\section{A BAYESIAN APPROACH TO META-ANALYSIS}

In carrying out a meta-analysis, one utilizes an estimated effect size $(z)$ and its squared standard error (known as the sampling variance; $s^{2}$ ) for each study. The main parameters of interest are the overall mean effect size $(\mu)$ and the variance $\left(\sigma^{2}\right)$ of the true effect sizes across studies. Two models that are commonly used in meta-analysis are the fixed-effect and random-effects models. The fixed-effect model assumes that all studies in the meta-analysis have a common (true) effect size $(6,29,31)$. That is, all factors that could moderate the effect size are assumed to be the same in every study; this is equivalent to assuming that $\sigma^{2}=0$. By contrast, a random-effects model postulates that whereas all studies share sufficient similarity to be included in a meta-analysis, they are not necessarily identical. The true effect size is therefore not exactly the same in all studies (i.e., $\sigma^{2}>0$ ), but effects from individual studies share a common distribution $(6,29,31)$. As noted by Ngugi et al. (18), the fixed-effect model is being replaced by the random-effects model in most disciplines because the heterogeneity of studies being combined is often such that the assumptions of the fixed-effect model cannot be justified. These models are not discussed further.

In a random-effects model, the observed effect size for the $i$ th study $(i=1, \ldots, k), z_{i}$, is given by the grand mean across studies $(\mu)$, a random deviation of the study's true effect from the mean $\left(u_{i}\right.$; which has a mean of 0 and an among-study variance of $\sigma^{2}$ ), and a random deviation within the study of the observed effect size from the true effect size for that study $\left(\varepsilon_{i}\right.$; with a mean of 0 and variance of $s_{i}^{2}$ [sampling variance]). The true effect size for the $i$ th study is then written as $\zeta_{i}=\mu+u_{i}$. The meta-analytical model can therefore be written as

$$
z_{i}=\zeta_{i}+\varepsilon_{i}
$$

$(6,16,31)$. The parameter $z_{i}$ therefore incorporates information on both among- and within-study variances, and a common distribution around $\mu$ is assumed.

In the Bayesian framework, equation 2 is often implemented as a hierarchical model that can be (partially) specified as

$$
\begin{aligned}
& z_{i}=\sim N\left[\zeta_{i}, s_{i}^{2}\right], \quad i=1, \ldots . . k \\
& \zeta_{i} \sim N\left[\mu, \sigma^{2}\right]
\end{aligned}
$$

Here, it is explicitly specified that the observed effect sizes for each study and the true effect size across studies have a normal distribution $(\mathrm{N}[]$,$) . Whereas this specification accords with$ classical models (16), there is no requirement for a normal distribution, and for both classical and Bayesian methods this assumption can be relaxed. Indeed, as we illustrate below, other distributions can be evaluated where appropriate. In the analysis, $\mu$ and $\sigma^{2}$ are estimated, and the $s_{i}{ }^{2}$ are held fixed (6). The key distinction between the classical and Bayesian model is that prior distributions are specified for the parameters $\mu$ and $\sigma^{2}$ in the Bayesian approach (or more generally, for any parameters that are in the hierarchical model). One can write this distributional property as

$$
\begin{aligned}
& \mu \sim-[-,-], \\
& \sigma^{2} \sim-[-,-]
\end{aligned}
$$

where $-[-,-]$ represents some (currently unspecified) prior distribution with parameters given in the brackets (see below). For a Bayesian analysis, therefore, equations 3 and 4 jointly define the model.
As indicated earlier, the choice of prior to be specified for $\mu$ and $\sigma^{2}$ can be controversial. However, it is reasonable to impose a noninformative prior for $\mu$, which can be done by specifying a normal distribution with a large variance. A noninformative prior can also be specified for $\sigma^{2}$ such as the gamma or inverse gamma, that confine $\sigma^{2}$ to positive values, although other distributions may be more appropriate. It is beyond the scope of this paper to illustrate the various types of potentially suitable priors, but Sutton and Abrams (29) provide good examples. Here, we illustrate a simple Bayesian meta-analysis using a series of studies that evaluated systemic acquired resistance (SAR) inducing compounds for control of fire blight (caused by Erwinia amylovora) in apple.

\section{CASE STUDY: BAYESIAN META-ANALYSIS OF SAR-INDUCING COMPOUNDS FOR CONTROL OF FIRE BLIGHT OF APPLE}

Database of SAR inducers. A review of efficacy trials evaluating products deemed to induce SAR was part of a larger metaanalysis of products evaluated for control of fire blight in the eastern United States (H. K. Ngugi, B. L. Lehman, and L. V. Madden, unpublished data). We illustrate a Bayesian meta-analysis with a subset of the data based on the antibiotic streptomycin, which is the standard treatment for fire blight control, and three SAR-inducing products; the gibberellic acid inhibitor prohexadione-calcium (Apogee), the harpin protein (Messenger), and acibenzolar-S-methyl (Actigard). Based on 58 studies conducted between 2000 and 2008 and published in Fungicide \& Nematicide Tests (F\&N Tests), Biological \& Cultural Tests (B\&C Tests), and Plant Disease Management Reports (PDMR), 79 independent treatments using streptomycin, 26 using Apogee, 7 using Actigard, and 13 using Messenger were summarized and used for the metaanalysis example in this paper. Hereafter, the treatments are considered independent studies. For a given treatment within a study, the response variable was the mean level of fire blight severity observed in plots receiving the antibiotic or the specific SARinducing compound. For each of the studies, the effect size was the response ratio $R$, computed as the mean fire blight severity of a treatment divided by the mean fire blight severity of the untreated check within the specific study. We used the log of $R[L=\ln (R)]$, which has better statistical properties and is preferred over $R$ when performing a meta-analysis $(10,19)$. A log response ratio below 0 indicates a positive percent control (disease reduction), and the lower the value of $L$, the better the observed control afforded by the treatment.

Example 1: Bayesian meta-analysis with noninformative priors and normal distribution. This is the simplest analysis that could be performed with Bayesian methods. We assume a random-effects model (equations 3 and 4), where the true effect sizes (measured in terms of $\ln (R)$ ) are from a normal distribution with mean $\mu$ and variance $\sigma^{2}$. This is the same assumption made in classical meta-analysis using a random-effects model (16). The model can be implemented using the Bayesian inference Using Gibbs Sampling software (WinBUGS; MRC Biostatistics Unit, Cambridge, UK), using the code below. The "model" statement corresponds to the two-level hierarchy described earlier by Madden and Paul (16) in their equations $8 \mathrm{a}$ and $8 \mathrm{~b}$ and is consistent with equation 3 above.

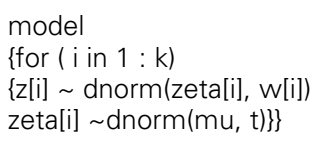

In this case, $z[\mathrm{i}]$ and zeta[i] correspond to $z_{i}$ and $\zeta_{i}$, respectively, in equation 3. w[i] is the weight for each study which is quantified using noninformative prior distributions; $w[i]$ is 
equivalent to $1 / s_{i}^{2}=s_{i}{ }^{-2}$. These weights are not altered in traditional analyses (Bayesian or otherwise). Moreover, $t$ in this code corresponds to $1 / \sigma^{2}=\sigma^{-2}$. In WinBUGS, one specifies the so-called precision of a distribution such as the normal (i.e., the inverse of its variance) rather than the variance directly. For example, dnormal $(0,0.0001)$ is the same as a normal distribution with mean 0 and variance $1 / 0.0001=100^{2}$.

For the noninformative prior distributions, we specify a normal distribution for $\mu$ (with a very large variance), and a gamma distribution for $\sigma^{-2}$ (with very large scale [variance]). This is written mathematically as

$$
\mu \sim \mathrm{N}\left[0,10^{6}\right] \text { and } \sigma^{-2} \sim \mathrm{G}\left[0.001,10^{3}\right]
$$

The choice of the values for the priors $\left(0,10^{6}\right.$, and $\left.0.001,10^{3}\right)$ is arbitrary here but such values are commonly used (31). Using the WinBUGS code, with the understanding that variances (or scale parameters) of distributions are given as precision values, one can write

$$
\begin{aligned}
& \mathrm{mu} \sim \operatorname{dnorm}(0.0,1.0 \mathrm{E}-6) \\
& \mathrm{t} \sim \text { dgamma(0.001, 0.001) } \\
& \text { sigsq }<-1 / \mathrm{t}
\end{aligned}
$$

where sigsq corresponds to $\sigma^{2}$. Specifically, note that the second 0.001 in the dgamma function (the precision of the inverse variance) is equivalent to the scale parameter of $10^{3}$ for the inverse variance above.

WinBUGS implements a Markov Chain Monte Carlo (MCMC) algorithm with a Gibbs sampler that calculates a parameter value conditional on the other parameters in the model and thus allows for the calculation of posterior distributions from a multidimensional parameter space. Simulations were performed with a total of 10,000 iterations. Usually, a number of initial iterations are disposed of as a "burn-in"; that is, an initial set of unstable parameter values are discarded so that they do not interfere with the calculation of the posterior parameter distribution. In all the analyses presented, the 5,000 initial iterations were disposed of.

The results of this analysis are presented in Table 2. Overall, the results are very similar to the classical meta-analysis, with minor differences. For example, the mean effect size estimated for Apogee using the classical approach is -0.56 with the $95 \%$ confidence interval (CI) of -0.72 to -0.37 , while the Bayesian analysis yields a mean of -0.54 with $95 \%$ credibility interval (CRI) of -0.75 to -0.33 (Table 2). In general, the CRIs from the Bayesian analyses were wider than the corresponding confidence intervals from the classical analysis. Presumably, this results from the Bayesian model allowing for full uncertainty in the estimation process (31), and "averaging over" the posterior distribution of the unknown among-study variance, whereas the classical approach calculates a single value of this statistic for each treatment. Nevertheless, a direct advantage of the Bayesian method is the fact that uncertainly related to these results can be easily quantified with the $95 \%$ CRI.

Example 2: Bayesian meta-analysis with noninformative priors and $\boldsymbol{t}$ distribution. When the sample size is small, such as in our example in which there are only seven studies that evaluated Actigard and 13 for Messenger, it is questionable whether the assumption of a normal distribution for the population of the effect sizes is valid. In such a case, a Student's $t$ rather than a normal distribution of the effect sizes may be a more appropriate assumption. The $t$ distribution is bell-shaped, but with smaller sample sizes it is less peaked than a standard normal distribution and with thicker tails. The variance is greater than 1 , but approaches 1 as the sample size increases. For $n>30$, the differences between the two distributions are negligible.

The code in WinBUGS for the random effects meta-analysis with a $t$ distribution is given below. This model is similar to the one implemented in example 1 except that now the effect sizes are assumed to be from a population with a $t$ distribution.

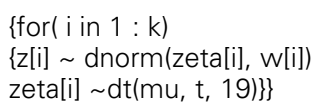

Here, the "19" indicates degree of freedoms and was arbitrarily chosen to be large so that Student's $t$ distribution is approximating a normal distribution.

As in example 1, we assume that we do not have any prior information for the mean of the population of the effect sizes, nor for the among-study variance of the true effect sizes. Therefore, we implement the same noninformative prior distributions as in example 1; that is, the WinBUGS code for the priors for mu and $t$ $\left(1 / \sigma^{2}\right)$ is the same as in example 1 . The results of this analysis are presented in Table 3. With the exception of Actigard, parameters for all other treatments are similar to the results obtained with a normal distribution as the prior. The mean and 95\% CRI values for Apogee based on the two distributions are almost identical, whereas those for streptomycin and Messenger are within the CRI values of either model (Table 3). Only seven studies evaluating the effects of Actigard on fire blight were available for use in this example. Specifying a Student's $t$ distribution resulted in a greater effect size for Actigard $(\mu=-0.40 ; 95 \%$ CRI $=-0.73$ to -0.10 ; Table 3) than was noted in the classical approach, for which the mean effect size for this product was not statistically significant (estimated $\mu$ : $-0.16,95 \%$ confidence interval: -0.44 to $0.13 ; P=$ 0.28; Table 1), or the Bayesian model with a normal distribution

\begin{tabular}{|c|c|c|c|c|c|c|}
\hline \multirow[b]{2}{*}{ Treatment } & \multicolumn{3}{|c|}{ Classical analysis ${ }^{v}$} & \multicolumn{3}{|c|}{ Bayesian analysisw $^{w}$} \\
\hline & Mean & $\mathbf{C l}^{\mathrm{x}}$ & $P>\chi^{2 y}$ & Mean & Median & CRI ${ }^{2}$ \\
\hline Streptomycin & -1.12 & $-1.31,-0.93$ & $<0.0001$ & -1.66 & -1.66 & $-2.11,-1.23$ \\
\hline Actigard & -0.16 & $-0.44,0.13$ & 0.280 & -0.22 & -0.21 & $-0.63,0.08$ \\
\hline Messenger & -0.27 & $-0.41,-0.13$ & 0.0002 & -0.38 & -0.38 & $-0.64,-0.11$ \\
\hline
\end{tabular}

TABLE 2

Mean effect sizes (log response ratio) and corresponding statistics for a classical and a Bayesian meta-analysis of three systemic acquired resistance-inducing treatments evaluated along with the antibiotic streptomycin for control of fire blight in apple 
(estimated $\mu$ : 0.22 ; 95\% CRI: -0.63 to 0.08 ; Table 1). Based on these results, it appears that available data (number of studies) for this treatment may not be sufficient and therefore the outcome, i.e., the posterior distribution, is "sensitive" to the choice of prior distribution.

Example 3: Bayesian meta-analysis with informative priors and normal distribution. The same code as in example 1 was used except that now informative priors were implemented. That is, we believe, based on published records (e.g., Maxson-Stein et al. [17]), that streptomycin is an effective treatment (i.e., the log response ratio of its mean effect size and confidence intervals should be negative). Mathematically this is quantified with a prior distribution with a small variance and a location parameter around the preconceived value of $\mu$. The shape of the prior distribution could also be changed, by selecting a model with a skewed shape or lighter tails, but this was not explored here. The informative prior was implemented in WinBUGS by replacing the code in example 1 for the distribution of $\mu$ with

$$
\begin{aligned}
& \mathrm{mu} \sim \operatorname{dnorm}(-3,0.1) \text { for streptomycin, and } \\
& \mathrm{mu} \sim \operatorname{dnorm}(-0.7,0.1) \text { for the SAR inducers }
\end{aligned}
$$

Specifically, a mean (on a log-ratio scale) of -3 was specified for streptomycin, and -0.7 for the other treatments; a variance of the prior distribution of $1 / 0.1=10$ was specified for all treatments (in contrast to example 1 , where a variance of $10^{3}$ was specified). The mean log-ratio -3 specified for streptomycin equates to $95 \%$ disease control, a realistic prior for a highly effective antibiotic for which 90 to $100 \%$ fire blight control on apple was previously reported in a study comparing its effectiveness with that of SAR inducers (17). The prior specified for SAR inducers $(-0.7)$ equates to $50 \%$ control and was based on the same study which reported $>50 \%$ fire blight control with Actigard in one of the 2 years the SAR-inducer was evaluated.

Figure 3 illustrates the posterior distributions of the effect sizes for four of the treatments (streptomycin, Apogee, Actigard, and Messenger) obtained from this analysis, indicating good convergence and parameter densities for models assuming a normal distribution with informative or noninformative priors. As in example 2, parameters for all treatments with the exception of Actigard are similar to those obtained with noninformative normal prior distribution (Table 3 ). The resulting posterior distributions for the two analyses are almost identical for streptomycin, Apogee, and Messenger (Fig. 3) indicating that with the number of studies available for analysis for these treatments, very informative priors did not have a large influence on the results. As is well known in Bayesian analysis $(12,29,31)$, informative priors have their largest impact with small data sets. This was supported here, where the results for Actigard clearly depended on the choice of prior (Table 3). Indeed, imposing an informative prior results in a posterior distribution that is different from that obtained with a noninformative prior for this treatment (Fig. 3F). This suggests that the direct evidence in the current investigation for this treatment was weak enough that the specified prior affected the final outcome. If the prior truly reflects prior beliefs, then a Bayesian would be willing to accept that Actigard is an effective control for fire blight. Such a result would be not evident from the classical meta-analysis. Here, the prior choice was more arbitrary (for demonstration purposes), so a strong outcome dependence on the prior raises questions about making inferences about the results for this one treatment based on a limited number of studies. These results also illustrate the power of Bayesian meta-analysis, e.g., that the consequences of a small sample size on the estimated effect size can be fully evaluated.

\section{BAYESIAN APPROACHES TO ADVANCED ISSUES IN META-ANALYSIS}

Incorporating heterogeneity and other distributions. Madden and Paul (16) discuss the issue of variability among studies that are incorporated in a meta-analysis. Among-study heterogeneity is of great interest especially in combining plant pathology studies because, with a few exceptions such as the uniform fungicide trials (UTFs) in the wheat scab (19-21) and soybean rust (25) pathosystems, most other meta-analyses involve studies carried out by different groups and often using disparate methods for which heterogeneity can be expected. The estimation $\sigma^{2}$ and incorporation of this parameter in the analysis is the primary way of accounting for heterogeneity. Madden and Paul (16) present different ways of characterizing heterogeneity among studies, and evaluating the impact of heterogeneity, from a classical approach. In Bayesian methods, starting with the data and a specified prior distribution for $\sigma^{2}$ (or for $\sigma^{-2}$ ), the heterogeneity between studies also can be modeled explicitly and its distribution determined using a hierarchical approach. This approach has been summarized by Berry (4), with examples from medical metaanalyses.

In one of the examples, Berry (4), reexamines the effects of among-study variability with Bayesian methods using studies that evaluated the outcomes of a cholesterol-lowering treatment on coronary death. In an earlier synthesis, Rossow et al. (23) derived conclusions on the effect of this treatment on coronary death with

TABLE 3

Mean effect sizes (log response ratio) and corresponding statistics for a Bayesian models with different distributions or with informative priors for the effect sizes of three systemic acquired resistance-inducing treatments evaluated along with the antibiotic streptomycin for control of fire blight in apple

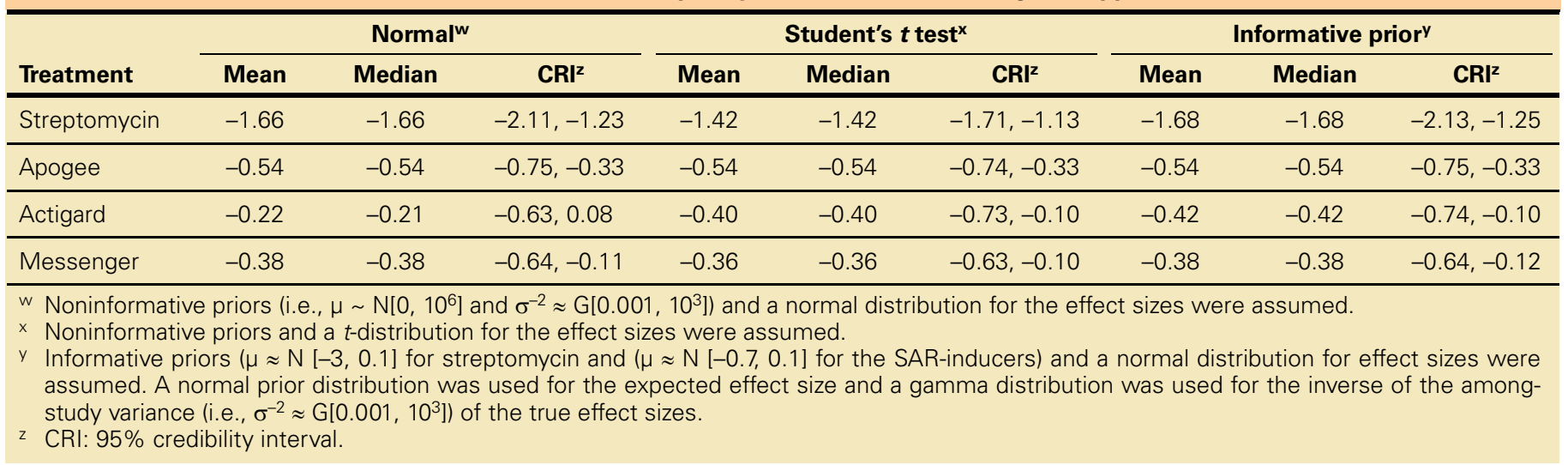


a classical meta-analysis of six independent trials. However, the results of this meta-analysis were not in agreement with the results of a much larger clinical study, conducted independently by a Scandinavian group to investigate the same issue (24). This large study involved 4,444 patients with coronary heart disease and studies were considered large if they involved over 1,000 patients (4). The meta-analysis example was based on six trials ( $i=1 \ldots 6$ ) (23). $X_{i}$ was the number of coronary deaths among $n_{i}$ patients in the control group for study $i$, and $Y_{i}$ the number of deaths among the $m_{i}$ patients in the treatment group. It was assumed that these variables had binomial distributions:

$$
X_{i} \sim \operatorname{binomial}\left(n_{i}, p_{i}\right) \text { and } Y_{i} \sim \operatorname{binomial}\left(m_{i}, q_{i}\right)
$$

where $p_{i}$ and $q_{i}$ are the probabilities of death in the two treatment groups.

At the second step of the hierarchy, $p_{i}$ and $q_{i}$ were analyzed with a log-linear model:

$$
\log \left[p_{i} /\left(1-p_{i}\right)\right]=\theta_{i} \text { and } \log \left[q_{i} /\left(1-q_{i}\right)\right]=\theta_{i}+\rho_{i}
$$

where $\theta_{i}$ is the $\log$ odds for each study. The variability in the $\theta_{i}$ represents the true control variation across studies. For example, the different diets of the subjects in the studies may partially explain the control variation across trials (4). The parameter $\rho_{i}$ represents the relationship between the control and the treatment for trial $i$ (essentially, a log odds ratio, i.e., the difference of the logits for the two treatments). For instance, the cholesterol treatment may affect subjects with different diets differently. This variation is captured by allowing $\rho_{\mathrm{i}} \mathrm{s}$ to vary across studies. Therefore, $\theta_{i}$ and $\rho_{i}$ are modeled as being conditionally independent and having a normal distribution. In the Bayesian framework, this is explicitly done by specifying distributions for the true $\theta_{i}$ and $\rho_{i}$ at this level of the hierarchy:

$$
\theta_{i} \sim \mathrm{N}\left[\mu_{\theta}, \sigma_{\theta}^{2}\right] \text { and } \rho_{i} \sim \mathrm{N}\left[\mu_{\rho}, \sigma_{\rho}^{2}\right]
$$

with prior distributions

$$
\begin{gathered}
\mu_{\theta} \sim \mathrm{N}[-1.5,0.25], \mu_{\rho} \sim \mathrm{N}[0,0.25] \\
\sigma_{\theta}{ }^{2} \sim \mathrm{IG}[3,3], \sigma_{\rho}{ }^{2} \sim \mathrm{IG}[3,3]
\end{gathered}
$$

Here, as mentioned above, IG represents the inverse-gamma distribution (equivalently, gamma distributions could have been specified for the inverse of these two variances). Informative priors were utilized here, based on the magnitude of the scale parameters used in the prior distributions.
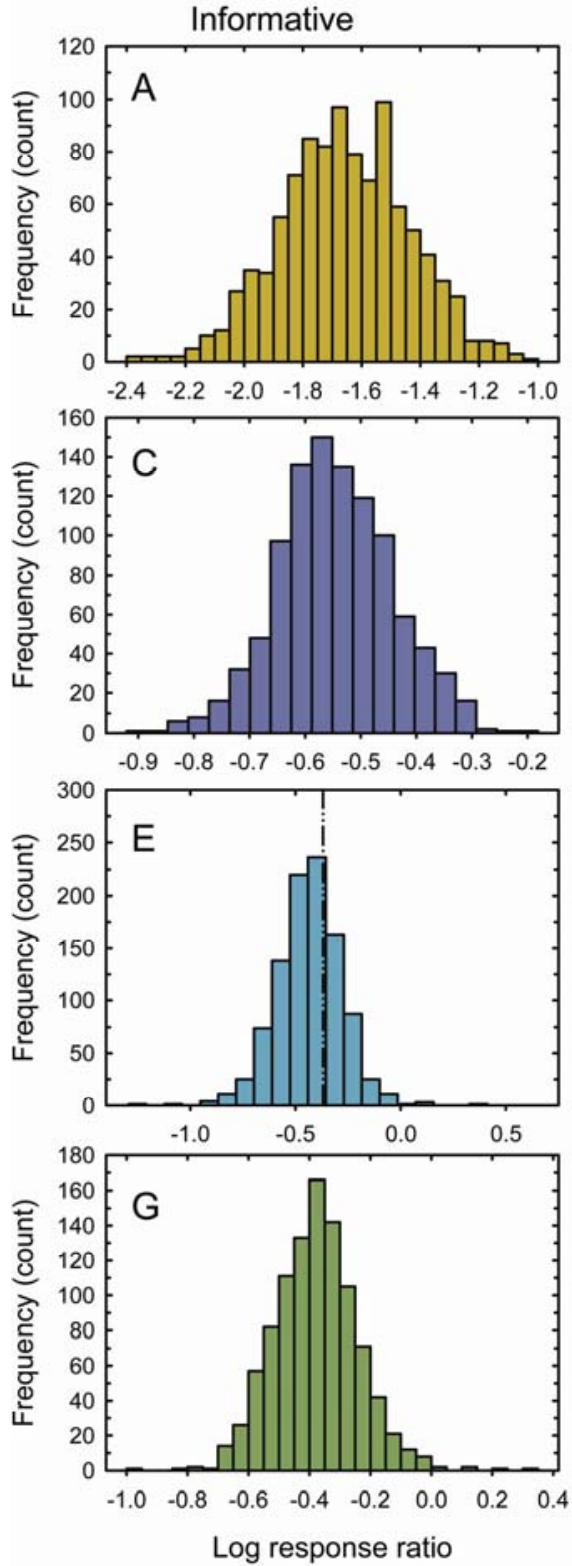

Log response ratio

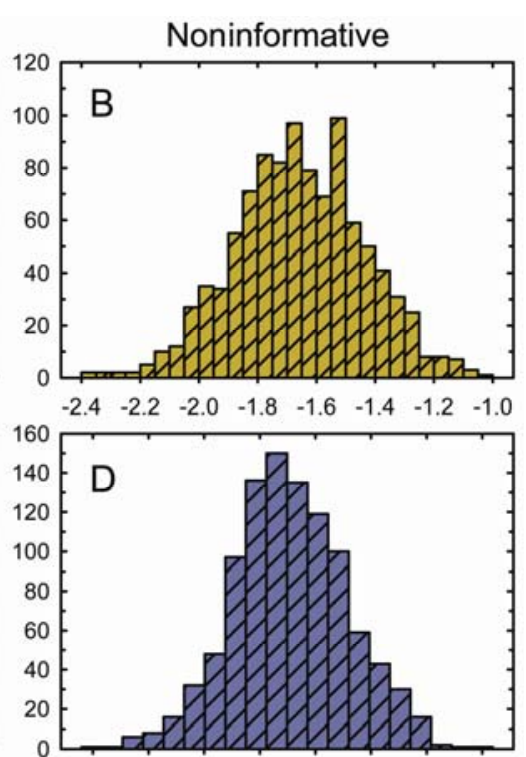

$\begin{array}{llllllll}-0.9 & -0.8 & -0.7 & -0.6 & -0.5 & -0.4 & -0.3 & -0.2\end{array}$
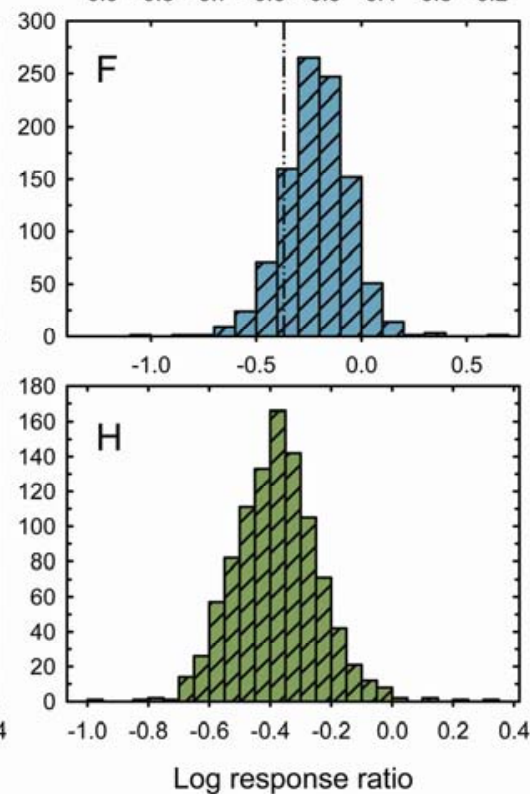

FIGURE 3

Posterior distribution of the effect sizes for $\mathbf{A}$ and $\mathbf{B}$, the antibiotic streptomycin, and the systemic acquired resistance-inducing products $\mathbf{C}$ and $\mathbf{D}$, Apogee, $\mathbf{E}$ and $\mathbf{F}$, Actigard, and $\mathbf{G}$ and $\mathbf{H}$, Messenger based on a Bayesian meta-analysis of 58 published studies that evaluated control of fire blight in apple in the eastern United States. Counts are for the last 1,001 values out of 5,000 simulations in a Bayesian analysis with a normal distribution and informative $(\mathbf{A}, \mathbf{C}, \mathbf{E}$, and $\mathbf{G})$ or noninformative (B, D, $\mathbf{F}$, and $\mathbf{H}$ ) priors for the effect size. Different scales are used for each of the product and the vertical line in $\mathbf{E}$ and $\mathbf{F}$ is set on the same position on the horizontal axis as a reference mark to illustrate the influence of an informative prior on the analysis for Actigard for which only a limited number of studies (seven) was available. 
As indicated above, the results from the classical meta-analysis of the six trials deviated significantly from the outcome of a large clinical trial conducted by the Scandinavian group (24) even though there was no reason to suggest that the latter study represented a different population from that of the meta-analysis. When studies were re-analyzed with Bayesian meta-analysis methods, results from the large independent study and the metaanalysis were in agreement. In the initial classical meta-analysis, the $\rho_{i}$ s were assumed constant and two of the six studies with observed $\rho_{i}$, smaller than that for the Scandinavian trial, contributed to a large extent to the deviation in results between the meta-analysis and the study conducted by the Scandinavian group. When the $\rho_{i}$ s were allowed to vary across trials with the Bayesian hierarchical methods outlined above, this discrepancy was corrected (4). The posterior mean $\rho$ and the 95\% CRI for the Scandinavian trial were shown to be within the posterior distribution of $\rho_{i}$ s for the six trials. Only by assuming that the $\rho_{i}$ s of the six trials were constant did the trial by the Scandinavian group appear to be distinctive (4). The use of hierarchical Bayesian models, therefore, not only explained the controversy, it resolved it!

Missing data. Studies with missing data are regularly encountered in meta-analysis. Bayesian methods for missing observations (estimated effect sizes or their sampling variances) include data augmentation and the use of hierarchical submodels (28). These models assume the missing data to be realizations of a random process with an underlying stochastic mechanism (i.e., a distribution model); that is, missing data are considered to form part of the distribution described in the model for which the parameters are to be estimated to (28), and because under the Bayesian framework the true values for parameters can be obtained (5), it is possible to compute the missing values. When using data augmentation, one imputes missing data from a variable's posterior distribution. For example, when the sampling variance (or standard error) is not reported in all studies the effect sizes cannot be weighted for those studies with missing variances. One potential solution, in addition to that described by Madden and Paul (16), to this problem is a fully Bayesian approach, in which pertinent external information is included $(1,2,27)$. Here, sampling variances $\left(s_{i}^{2}\right)$ are not considered fixed values, as they were in the examples above, but rather observations from a distribution of values, which can accommodate the lack of variances from some of the studies. Commonly, the IG prior distribution is used for $s_{i}^{2}$ because it has the advantage to constrain variance values to greater than zero (as a variance must be) and can accommodate informative a priori beliefs (2). This aspect is also important because there is often evidence about the level of variability in a population that cannot be incorporated formally into the meta-analysis otherwise.

Incorporating information to increase precision in metaanalysis with small number of studies. When conducting a metaanalysis of plant pathology studies, one is likely to encounter cases where the question of interest has only been addressed in a limited number of studies. For example, in the initial stages of product testing, the efficacy of a new fungicide or biocontrol agent may only be evaluated in a limited number of studies. Large heterogeneity among studies in the true effect sizes (large $\sigma^{2}$ ) may be a problem in such situations. In many cases, there is information from sources other than the trials under consideration that is relevant to the treatment comparison of interest. In a Bayesian approach, such information may be incorporated into the metaanalysis through the specified priors to improve precision in the estimation of both heterogeneity and relative efficacy.

Higgins and Whitehead (12) discuss this issue, provide details of the theoretical background, and illustrate how to deal with a limited number of studies with a medical example from betablockers and/or sclerotherapy treatments in the prevention of first bleeding in cirrhosis. Nine of the trials made a comparison between beta-blockers and a control treatment and 19 trials compared sclerotherapy and a control. Two trials compared all three treatments, and consequently only these two trials made a direct comparison between beta-blockers and sclerotherapy. The authors were interested in the differences between beta-blockers and sclerotherapy, although in the initial studies both treatments were significantly different from the placebo. With only two studies, precision of an estimated expected effect size will be low if heterogeneity is high between the studies, resulting in low statistical power (Madden and Paul [16]). The authors implemented a Bayesian analysis using the Gibbs sampler with a noninformative prior distribution for $\sigma^{2}$ (i.e., $\operatorname{IG}\left[10^{-3}, 10^{-3}\right]$ ). However, this did not give a satisfactorily convergent chain, presumably because there was little information regarding heterogeneity between the trials.

If a meta-analysis of such a small number of studies is to be performed, imposing an influential prior distribution (i.e., one with a small variance) may be critical to accurately incorporate the limited experience into the posterior probability. Higgins and Whitehead (12) derived the influential prior from the studies conducted previously that compared each treatment separately with a placebo. Specifically, they assumed that the distribution for $\sigma^{2}$ in both beta-blocker and the sclerotherapy studies was an IG, as in the noninformative analysis that failed to converge, but in this case the precision for the distribution was changed from $\left(10^{-3}, 10^{-3}\right)$ to the more influential $(1.0,0.35)$. By imposing the influential prior the Bayesian model converged and passed diagnostic tests. Moreover, the results showed that there was no evidence that the true heterogeneity parameters should be different for the betablocker and the sclerotherapy treatments.

Publication bias. Often it is useful for the data analyst to address the possible presence of publication bias, and Madden and Paul (16) provide details of such an analysis based on classical (frequentist) methods. One approach that has been used in Bayesian analysis is to treat the studies in question as if they were missing data and to employ the data argumentation techniques described above. For example, Givens et al. (9) used a Bayesian hierarchical model for meta-analysis that combines the estimated effect sizes from heterogeneous individual studies after estimating and adjusting for potential publication bias. They used a dataaugmentation technique, with the assumption that studies are missing with probabilities that are a function of their lack of statistical significance. They implemented this model on a set of 31 studies that the Environmental Protection Agency (EPA) used to evaluate the effects of passive smoking, or exposure to environmental tobacco smoke (ETS). In 1992, EPA issued a report concluding that ETS is a class A human carcinogen. This was based largely on an argument by analogy with data on the relationship between lung cancer and active smoking, but also included a meta-analysis of 31 studies on the association of lung cancer in patients who had never smoked but were subject to ETS exposure through spousal smoking. Givens et al. (9) estimated that in the United States, some four or five studies were missing and when they accounted for the effect of these studies applying a hierarchical Bayesian model, the overall relative risk estimate decreased from 1.17, with a $95 \%$ posterior CRI of 1.02 to 1.33 to 1.10 , with a $95 \%$ interval of 0.95 to 1.29 . In a later effort, the same authors expanded the original hierarchical Bayesian model to account for multiple "tiers" that refer to groups defined based on the quality classifications of the studies (26). It is possible to see a case where such an approach can be taken in plant pathology, where studies are not used in a meta-analysis for lack of measures of variation. This is especially the case for metaanalyses based on product efficacy trials which report mean separations but not a measure of within-study variability. 


\section{SOFTWARE AVAILABILITY}

For many years the principles of Bayesian statistics have been well understood. However, implementation in areas such as metaanalysis has been slow until recently because of computational complexity. Fortunately, many of the algorithms for performing Bayesian analysis have recently become available online. Most of them are contained in StatLib, a system for the electronic distribution of software, data sets, and other information that can be accessed via the Statistics Department at Carnegie Mellon University. The most well-developed and widely used software for Bayesian analysis is WinBUGS (15), which executes MCMC algorithms with a Gibbs sample, and is freely available online from the Biostatistics Unit of the Medical Research Council of Cambridge, UK. Table 4 lists some statistical software capable of Bayesian analysis either by implementing MCMC algorithms with a Gibbs sampler directly or by invoking WinBugs.

\section{CONCLUSIONS}

Although Bayesian methods for combining and analyzing results from independent studies are widely accepted and commonly used in other fields $(3,7,30)$, they have yet to be used in plant pathology in any major way. There are a number of potential advantages in using Bayesian statistics for meta-analysis. Firstly, Bayesian methods enable an analysis to take account of parameter uncertainty, a feature that classical (frequentist) methods have until recently been lacking. Secondly, Bayesian approaches enable pertinent background information to be included in a metaanalysis through the specification of informative prior distributions, which otherwise would be ignored. A third advantage of the use of Bayesian methods occurs when there are a number of potentially competing models that need to be evaluated. Adopting a classical approach often entails choosing between models (e.g., fixed and random-effects), which therefore fails to account for the inherent model uncertainly. By contrast, Bayesian methods allow for a full evaluation of all competing models $(29,31)$. Bayesian methods would also be very useful in dealing with studies for which an estimate of within-study variance (needed to weight the estimated effect sizes) was lacking. In this paper, we have illustrated several Bayesian meta-analysis procedures to illustrate what is possible. Given the power of Bayesian methods to address most of the issues encountered in meta-analysis, we would argue that its application for the synthesis of plant pathology studies is long overdue.

\section{ACKNOWLEDGMENTS}

We thank L. V. Madden and H. Scherm for critical reviews that greatly improved this paper. H. K. Ngugi was supported by funds from the State Horticultural Association of Pennsylvania (SHAP).

\section{LITERATURE CITED}

1. Abrams, K. R., Gillies, C. L., and Lambert, P. C. 2005. Meta-analysis of heterogeneously reported trials assessing change from baseline. Stat. Med. 24:3823-3844.

2. Abrams, K. R., Lambert, P. C., Sanso, B., and Shaw, C. 2000. Metaanalysis of heterogeneously reported study results: A Bayesian approach. Pages 29-64 in: Meta-Analysis in Medicine and Health Policy. D. K. Stangl and D. A. Berry, eds. Marcel Dekker, Inc., New York.

3. Ashby, D. 2006. Bayesian statistics in medicine: A 25 year review. Stat. Med. 25:3589-3631.

4. Berry, S. M. 2000. Meta-analysis versus large trials: Resolving the controversy. Pages 65-81 in: Meta-Analysis in Medicine and Health Policy. D. K. Stangl and D. A. Berry, eds. Marcel Dekker, Inc., New York.

5. Bolstad, W. M. 2007. Introduction to Bayesian Statistics. 2nd ed. Wiley, Hoboken, NJ.

6. Borenstein, M., Hedges, L. V., Higgins, J. P. T., and Rothstein, H. R. 2009. Introduction to Meta-Analysis. John Wiley \& Sons Ltd., West Sussex, U.K.

7. Choy, S. L., O’Leary, R., and Mengersen, K. 2009. Elicitation by design in ecology: Using expert opinion to inform priors for Bayesian statistical models. Ecology 90:265-277.

8. Gelman, A., Carlin, J. B., Stern, H. S., and Rubin, D. B. 1996. Bayesian Data Analysis. Chapman \& Hall/CRC, New York.

9. Givens, G. H., Smith, D. D., and Tweedie, R. L. 1997. Publication bias in meta-analysis: A Bayesian data-augmentation approach to account for issues exemplified in the passive smoking debate. Stat. Sci. 12:221-250.

10. Hedges, L. V., Gurevitch, J., and Curtis, P. S. 1999. The meta-analysis of response ratios in experimental ecology. Ecology 80:1150-1156.

11. Higgins, J. P. T., and Spiegelhalter, D. J. 2002. Being skeptical about meta-analysis: A Bayesian perspective on magnesium trials in myocardial infarction. Intern. J. Epidemiol. 31:96-104.

12. Higgins, J. P. T., and Whitehead, A. 1996. Borrowing strength from external trials in a meta-analysis. Stat. Med. 15:2733-2749.

13. Light, L., and Pillemer, D. 1984. Summing Up: The Science of Reviewing Research. Harvard University Press. Cambridge, MA.

14. Liu, J. S., and Lawrence, C. E. 1999. Bayesian inference on biopolymer models. Bioinformatics 15:38-52.

15. Lunn, D. J., Thomas, A., Best, N., and Spiegelhalter, D. 2000. WinBUGS-A Bayesian modelling framework: Concepts, structure, and extensibility. Stat. Comp. 10:325-337.

16. Madden, L. V., and Paul, P. A. 2011. Meta-analysis for evidence synthesis in plant pathology: An overview. Phytopathology 101:16-30.

17. Maxson-Stein, K., He, S.-Y., Hammerschmidt, R., and Jones, A. L. 2002. Effect of treating apple trees with acibenzolar-S-methyl on fire blight and expression of pathogenesis-related protein genes. Plant Dis. 86:785-790.

\begin{tabular}{|c|c|c|}
\hline $\begin{array}{l}\text { WinBUGS (Bayesian Inference Using Gibbs } \\
\text { Sampling [15]) }\end{array}$ & $\begin{array}{l}\text { Biostatistics Unit of the Medical Research Council } \\
\text { of Cambridge, UK (www.mrc-bsu.cam.ac.uk) }\end{array}$ & $\begin{array}{l}\text { The most well-developed and widely used } \\
\text { software for Bayesian analysis. Available } \\
\text { freely online. }\end{array}$ \\
\hline SAS (Statistical Analysis System) & (SAS Institute, Cary, NC) & $\begin{array}{l}\text { New Bayesian capabilities through the } \\
\text { MCMC procedure. Capable of running } \\
\text { WinBugs through open-source macros. }\end{array}$ \\
\hline $\mathrm{R}$ & $\begin{array}{l}\text { R Foundation for Statistical Computing, Vienna, } \\
\text { Austria }\end{array}$ & $\begin{array}{l}\text { Has Bayesian capabilities by implementing } \\
\text { WinBugs. }\end{array}$ \\
\hline
\end{tabular}




\section{Phytopathology}

18. Ngugi, H. K., Esker, P. D., and Scherm, H. 2011. Meta-analysis to determine the effects of plant disease management measures: Review and case studies on soybean and apple. Phytopathology 101:31-41.

19. Paul, P. A., Lipps, P. E., Hershman, D. E., McMullen, M. P., Draper, M. A., and Madden, L. V. 2007. A quantitative review of tebuconazole effect on Fusarium head blight and deoxynivalenol content in wheat. Phytopathology 97:211-220.

20. Paul, P. A., Lipps, P. E., Hershman, D. E., McMullen, M. P., Draper, M. A., and Madden, L. V. 2008. Efficacy of triazole-based fungicides for Fusarium head blight and deoxynivalenol control in wheat: A multivariate meta-analysis. Phytopathology 98:999-1011.

21. Paul, P. A., Lipps, P. E., and Madden, L. V. 2006. Meta-analysis of regression coefficients for the relationship between Fusarium head blight and deoxynivalenol content of wheat. Phytopathology 96:951-961.

22. Redmond, C. K., and Colton, T. 2001. Biostatistics in Clinical Trials. John Wiley \& Sons, Inc., New York.

23. Rossow, J. E., Lewis, B., and Rifkind, B. M. 1990. The value of lowering cholesterol after myocardial infarction. N. Engl. J. Med. 323:1112-1119.

24. Scandinavian Simvastatin Survival Trial Group. 1994. Randomised trial of cholesterol lowering in 4444 patients with coronary heart disease: The
Scandinavian Simvastatin Survival Study (4S). Lancet 344:1383-1389.

25. Scherm, H., Christiano, R. S. C., Esker, P. D., Del Ponte, E. M., and Godoy, C. V. 2009. Quantitative review of fungicide efficacy trials for managing soybean rust in Brazil. Crop Prot. 28:774-782.

26. Smith, D. D., Givens, G. H., and Tweedie, R. L. 2000. Adjustment for publication bias and quality bias in Bayesian meta-analysis. Pages 277304 in: Meta-Analysis in Medicine and Health Policy. D. K. Stangl and D. A. Berry, eds. Marcel Dekker, Inc., New York.

27. Spiegelhalter, D. J., Abrams, K. R., and Myles, J. P. 2003. Bayesian Approaches to Clinical Trials and Healthcare Evaluation. John Wiley \& Sons, Chichester, U.K.

28. Stangl, D. K., and Berry, D. A. 2000. Meta-analysis: Past and present challenges. Pages 1-28 in: Meta-Analysis in Medicine and Health Policy. D. K. Stangl and D. A. Berry, eds. Marcel Dekker, Inc., New York.

29. Sutton, A. J., and Abrams, K. R. 2001. Bayesian methods in meta-analysis and evidence synthesis. Stat. Methods Med. Res. 10:277-303.

30. Sutton, A. J., and Higgins, J. P. T. 2008. Recent developments in metaanalysis. Stat. Med. 27:625-650

31. Whitehead, A. 2002. Meta-Analysis of Controlled Clinical Trials. John Wiley \& Sons, West Sussex, England. 